\title{
NEOADJUVANT TRANSCATHETER ARTERIAL CHEMOEMBOLIZATION FOR BILIARY TUMOR THROMBOSIS: A RETROSPECTIVE STUDY
}

Yangyang Shen

Shandong Cancer Hospital affiliated to Shandong University, Shandong Academy of Medical

Sciences

Pang Li

Department of General Surgery, Lianyuan People's Hospital of Hunan Province

Kai Cui, Zhendan Wang

Shandong Cancer Hospital affiliated to Shandong University, Shandong Academy of Medical

Sciences

Fachang $Y_{U}$

Department of Internal Medicine, Ji'nan Fifth People's Hospital

\author{
He Tian \\ Shandong Cancer Hospital affiliated to Shandong University, Shandong Academy of Medical \\ Sciences \\ syyvly@126.com \\ Sheng li \\ Shandong Cancer Hospital affiliated to Shandong University, Shandong Academy of Medical \\ Sciences \\ Shandong Provincial Collaborative Innovation Center for Neurodegenerative Disorders, Qingdao \\ University \\ sdywyiy@126.com
}

Objectives: Curative hepatectomy and tumor thrombectomy for hepatocellular carcinoma with complicating biliary tumor thrombosis (HCC/BTT) is associated with high surgical morbidity and mortality. This retrospective study evaluated the effectiveness and safety of neoadjuvant transcatheter arterial chemoembolization (TACE) in HCC/BTT patients scheduled for curative resection.

Methods: Thirty consecutive patients with diagnosed HCC/BTT were hospitalized for neoadiuvant TACE and elective curative liver resection (group A; $n=20$ ) or curative liver resection alone (group $B ; n=10$ ). The primary outcome measure was median survival.

Results: Group A had a significantly shorter overall operative time $(160 \pm 25$ versus $190 \pm 35 \mathrm{~min} ; p<.01)$ and duration of inflow control $(14.3 \pm 3.6$ versus $25.1 \pm 5.1$ $\min ; p<.01)$ and significantly less intraoperative blood loss ( $150 \pm 35$ versus $520 \pm 75 \mathrm{ml} ; p<.01)$ and transfusion $(100 \pm 40$ versus $375 \pm 55 \mathrm{ml} ; p<.01)$ as compared to group B. Among patients undergoing both thrombectomy and curative resection, the median survival of group $A$ was significantly longer than that of group $B$ (28.5 [9-54] versus $21.5[6-39]$ months; $p<.01$ ); among those who received thrombectomy alone, the median survival of group A was also significantly longer than that of group B (12.8 [6-25] versus $4.5[2-7]$ months; $p<.01)$.

Conclusions: Neoadjuvant TACE significantly reduced the surgical risk of curative liver resection and significantly prolonged median survival in HCC patients with complicating BTT.

Keywords: Hepatocellular carcinoma, Biliary tumor thrombosis, Neoadjuvant therapy, Curative resection, Thrombectomy, Survival

Primary hepatocellular carcinoma (HCC) is a highly invasive tumor, likely to infiltrate the portal vein, hepatic veins, and even the inferior vena cava (1). Vascular invasion is a welldocumented factor of poor prognosis in HCC patients (2). However, involvement of the bile duct, namely, biliary tumor thrombosis (BTT), is less frequently encountered in surgical practice (3), usually identified in 1.2-9 percent of HCC patients during curative hepatectomy or autopsy (4). Biliary invasion may result from tumor infiltration or migration and frequently extends to the hilum, contralateral intrahepatic bile duct, or the common bile duct (5). This condition consequently results in obstructive jaundice and worsens the pre-existing liver dysfunction of HCC

Drs. Shen and Pang Li contributed equally to this work. This study was financially supported by a grant from the Natural Science Foundation of Shandong (No. Y2008C34). The authors declare no conflict of interest. patients. However, the presence of a tumor thrombus is often concealed by underlying chronic hepatitis or liver cirrhosis.

Although biliary invasion may occur at an early stage, HCC with complicating BTT (HCC/BTT) is generally believed to be ineligible for curative treatment (6). The overall survival of HCC/BTT patients at 1 and 3 years (62 percent and 30 percent, respectively) is poor compared with those free of BTT ( 89 percent and 73 percent) (7), and the 1- and 3-year cumulative survival of HCC/BTT patients with concomitant portal or hepatic venous tumor thrombosis (43 percent and 17 percent, respectively) is even poorer than that of patients without concomitant venous tumor thrombosis ( 89 percent and 52 percent) (7).

Curative hepatectomy with tumor thrombectomy is the mainstay definitive treatment for HCC/BTT $(8 ; 9)$. However, curative liver resection is associated with a higher risk of surgical morbidity and mortality in jaundiced HCC patients, due to aggravated liver dysfunction (10). The outcomes of curative 
hepatectomy remain variable and less than satisfactory, with a reported 3-year survival rate of 10 percent to 47 percent, depending on the presence of concomitant vascular involvement (7).

Transcatheter arterial chemoembolization (TACE) is a minimally invasive therapeutic technique used as palliative treatment alone or converting/neoadjuvant therapy before curative hepatectomy (11). TACE is also associated with a promising survival benefit for patients with well-preserved liver function (11). Preoperative TACE was reported to minimize surgical morbidity and mortality and improve overall survival after curative liver resection in selected HCC patients with major vascular invasion (12). The present retrospective study aimed to evaluate the effectiveness and safety of preoperative TACE for HCC/BTT patients scheduled for curative resection.

\section{METHODOLOGY}

\section{Study Protocol}

From January 1996 to October 2011, a total of 1,352 patients were diagnosed with $\mathrm{HCC}$ based on liver imaging and serum alpha-fetoprotein (AFP) at our hepatobiliary surgery unit. Of these patients, 132 were HCC/BTT, and thirty of these underwent curative liver resection with or without neoadjuvant TACE. An observational and retrospective study was performed by analyzing the 30 consecutive $\mathrm{HCC} / \mathrm{BTT}$ patients who underwent neoadjuvant TACE and curative liver resection (Group A; $n=20$ ) or curative liver resection alone (Group B; $n=10)$.

The following inclusion criteria were used: with concomitant BTT on preoperative imaging; with a liver function reserve of Child-Pugh class A or B on admission; with a total serum bilirubin level $<500 \mu \mathrm{mol} / \mathrm{L}$; and eligible for TACE and curative resection. The exclusion criteria were as follows: with any extrahepatic metastases or extensive portal vein involvement; with pre-existing serious cardiovascular or cerebrovascular disease, hepatorenal dysfunction, or complicating diabetes mellitus; or rejecting curative resection.

The patients were fully informed of their conditions, and decisions regarding the treatment options of neoadjuvant TACE and curative liver resection or curative liver resection alone were made by the patients. The procedures contributing to this work comply with the ethical standards of the relevant national and institutional committees on human experimentation and with the Helsinki Declaration of 1975, as revised in 2008. The Institutional Review Board at Shandong Provincial Hospital and Shandong Cancer Hospital approved the study protocol.

\section{Preoperative Workup}

Abdominal contrast-enhanced computed tomography was performed to characterize the liver tumor and exclude any radiologically detectable extrahepatic metastases. The classification of BTT was previously described by Ueda et al. (13): type I, involving left or right hepatic duct but not the biliary convergence, and usually not jaundiced; type II, involving left or right hepatic duct as well as the biliary convergence, and usually jaundiced; type III, involving common hepatic duct or common bile duct, and jaundiced to various extent; and type IV, involving bilateral hepatic ducts as well as intrahepatic and extrahepatic bile ducts.

\section{TACE and Surgical Procedures}

Superselected TACE was performed through the right femoral artery using the Seldinger technique, and chemoembolizing agents included doxorubicin, fluorouracil, camptothecin, iodinated oil, and gelatin sponge. Tumor response to TACE was evaluated using contrast-enhanced computed tomography scan at post-TACE 2 weeks. A patient was eligible for further curative hepatectomy 2 weeks after TACE if the retention rate of iodocyanine green at 15 minutes was less than 10 percent on preoperative assessment and expected to be $\geq 30$ percent of the total liver volume after resection.

Primary $\mathrm{HCC}$ was removed using the standard anatomical en bloc resection technique. BTT was removed using forceps for BTT involving hepatic ducts and interlobular bile ducts on the stump, exploration and thrombectomy of the extrahepatic bile duct, or a combination of these two techniques. Cholangiostomy was performed to antegradely explore the common bile duct to the bile duct stump on the resection surface. The common bile duct was thoroughly rinsed with sterile water to completely remove BTT. The biliary T-tube was maintained for 2 weeks and removed when the follow-up cholangiography determined that there was no BTT residual.

All patients were followed up at outpatient clinics using routine hematologic, biochemical, and serum AFP assays and liver imaging, every 6 months within the first postoperative year and every 12 months afterward.

\section{Outcome Measures}

The primary outcome measure was the median overall survival after the surgical treatment. The secondary outcome measures were the median operative time, median volume of intraoperative blood loss, postoperative recovery times, surgical morbidities, and tumor recurrence.

\section{Statistical Analysis}

All continuous data were expressed as mean \pm standard deviation. Differences between the two groups were compared using two independent samples Student $t$-test. All categorical data were expressed as $n(\%)$ and compared using the Fisher exact probability test. Survival in relation to the procedure received (TACE plus surgery versus surgery alone) were estimated with the Kaplan-Meier curve and compared using the log rank test. A $p$-value $<.05$ was considered statistically significant. 


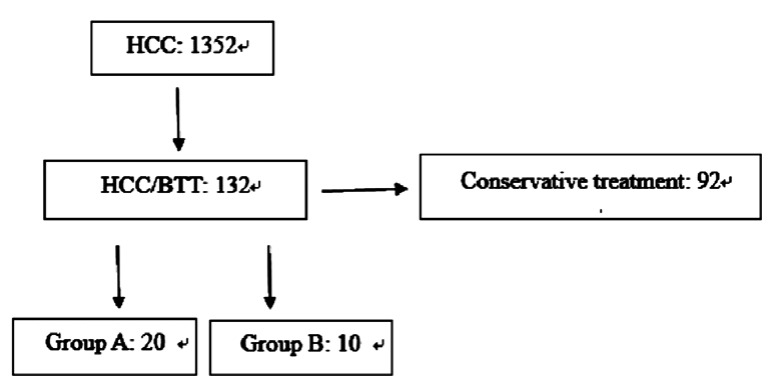

Figure 1. Of 1,352 patients diagnosed with primary $\mathrm{HCC}$ at the hepatobiliary surgery unit, 132 patients were $\mathrm{HCC} / \mathrm{BIT}$, from which thirty patients who underwent curative liver resection with or without neoadjuvant TACE were categorized into Group A (neoadjuvant TACE and curative liver resection $(n=20)$ and Group B (curative liver resection alone; $n=10$ ).

\section{RESULTS}

\section{Patient Characteristics}

The patient assignment flow chart is shown in Figure 1. Of 1,352 patients with primary HCC, concomitant BTT was identified in 132 (9.8 percent). Among these $132 \mathrm{HCC} / \mathrm{BTT}$ patients, thirty patients ( 22.7 percent) were determined to be eligible for curative resection (Table 1). The tumor thrombi were located in the common bile duct $(n=5)$, right hepatic duct $(n=11)$, left hepatic duct $(n=13)$, and caudate lobe $(n=1)$. BTT was classified as type I, II, III, and V in four, twelve, nine, and five patients, respectively. Bile duct dilation occurred concomitantly in twenty-five patients (80.0 percent).

Among the thirty HCC/BTT patients, twenty (66.7 percent) patients underwent preoperative TACE and subsequent surgical treatment (group A), and ten (33.3 percent) patents underwent surgical treatment alone (group B). The two groups were comparable in terms of age, gender, body mass index, number, size and location of HCC, location and type of BTT, serum AFP level, liver function reserve, and concomitant medical and surgical conditions (all $p$-values $>.05$ ).

\section{TACE Data}

For all patients in group A, TACE was completed successfully and uneventfully with a single attempt. No post-TACE infection, bleeding, liver dysfunction, or post-embolization syndrome occurred in any of these patients, and the follow-up liver function test at 2 weeks showed a liver function reserve of Child-Pugh class A or B.

\section{Operative Data}

In group A, BTT was adjacent to or separated from the liver disease in three (15.0 percent) and seventeen ( 85.0 percent) patients, respectively. The bile duct wall was intact in twelve (60.0 percent) patients and involved in eight ( 40.0 percent) patients. In group B, biliary tumor thrombi were in the shape of a bile duct cast, adjacent to or separated from the primary cancer in one (10.0 percent) and nine (90.0 percent) patients, respectively. The tumor thrombi were highly mobile in six $(60.0$ percent) patients on dissection, whereas the invasion of tumor thrombus into the bile duct wall was identified in four (40.0 percent) patients.

Operative and postoperative recovery outcomes are shown in Table 2. The rate of curative liver resection in group A was similar to that of group B (60.0 percent [12/20] versus 70.0 percent $[7 / 10] ; p>0.05)$; and that of tumor thrombectomy alone in group A was similar to that of group B (40.0 percent [8/20] versus 30.0 percent $[3 / 10] ; p>.05)$. The two groups underwent similar liver resection: tumor thrombi and liver tumor were resected in an en bloc manner in 3 patients of group A and in 1 patient of group B, respectively. Overall operative time was significantly shorter in group A than in group B (160 \pm 25 versus $190 \pm 35 \mathrm{~min}, p<.05)$. Duration of inflow control was also significantly shorter in group A than in group B (14.3 \pm 3.6 versus $25.1 \pm 5.1 \mathrm{~min} ; p<.01)$. Moreover, intraoperative blood loss $(150 \pm 35$ versus $520 \pm 75 \mathrm{ml} ; p<.01)$ and transfusion $(100 \pm 40$ versus $375 \pm 55 \mathrm{ml} ; p<.01)$ were significantly less in group A than in group B.

No massive hemobilia occurred in group A, whereas massive bleeding occurred in 4 patients of group B (4/10; 40.0 percent) with bile duct wall involvement on tumor thrombectomy; bleeding was successfully controlled by placing a large-caliber T-tube to interrupt the hepatic artery inflow. Postoperative recovery results with respect to volume of peritoneal drainage, time to remove peritoneal drain, and time to resume anal passage were similar between the two groups. However, preoperative TACE was associated with a slightly, although not statistically significant, shorter hospitalization $(19.2 \pm 3.2$ versus $24.3 \pm 2.9 \mathrm{~d}, p>.05)$.

No postoperative complications, such as bile leakage, hemobilia, liver dysfunction, intra-abdominal infection, or mortality occurred in any patient, except for pleural effusion $(n=1)$, pulmonary infection $(n=1)$, and wound fat liquefaction $(n=1)$. All these complications were successfully managed with symptomatic treatment. Obstructive jaundice resolved 3 weeks after thrombectomy, and the liver function test returned to normal in all patients.

\section{Pathological and Follow-up Outcomes}

Pathological examination confirmed the diagnosis of HCC, and all resection specimens had a clean margin. The tumor thrombi appeared to be pale, with crab-like strands or columns mixed with yellowish or brownish blood clots on gross examination. The histology of tumor thrombi showed an irregular mass of HCC cells interwoven with red blood cells, white blood cells, or cellular debris.

All patients were followed up for 6-54 months (median, 20.2 months) as scheduled, and no patients were lost to followup until the time of drafting this manuscript. For patients undergoing thrombectomy and curative resection, group A had a significantly longer median overall survival than group 
Table 1. Baseline Characteristics of HCC/BTT $(n=30)$

\begin{tabular}{|c|c|c|c|c|}
\hline & & Group A & Group B & $p$-Value \\
\hline $\begin{array}{l}\text { Age, years } \\
\text { Gender, } M / F \\
\text { Body mass index, } \mathrm{kg} / \mathrm{m}^{2}\end{array}$ & & $\begin{array}{l}54.3 \pm 5.6 \\
13 / 7 \\
23.6 \pm 0.9\end{array}$ & $\begin{array}{l}53.0 \pm 6.0 \\
7 / 3 \\
24.1 \pm 0.2\end{array}$ & $\begin{array}{l}0.563 \\
1.000 \\
0.532\end{array}$ \\
\hline Underlying liver conditions, $n(\%)$ & $\begin{array}{l}\text { Chronic hepatitis } \\
\text { Liver cirrhosis } \\
\text { Liver steatosis }\end{array}$ & $\begin{array}{l}19(95.0) \\
18(90.0) \\
1(5.0)\end{array}$ & $\begin{array}{l}10(100.0) \\
10(100.0) \\
0(0.0)\end{array}$ & $\begin{array}{l}1.000 \\
1.000 \\
1.000\end{array}$ \\
\hline Liver function reserve, $n(\%)$ & $\begin{array}{l}\text { Child-Pugh class A } \\
\text { Child-Pugh class B }\end{array}$ & $\begin{array}{l}17(85.0) \\
3(15.0)\end{array}$ & $\begin{array}{l}8(80.0) \\
2(20.0)\end{array}$ & 1.000 \\
\hline ICG 15,\% & & $24 \pm 11$ & $27 \pm 10$ & 0.476 \\
\hline Laboratory values & $\begin{array}{l}\text { Hemoglobin, } \mathrm{g} / \mathrm{dL} \\
\text { Platelet count, } 10^{9} / \mathrm{L} \\
\text { Albumin, } \mathrm{g} / \mathrm{L} \\
\text { Aspartate aminotransferase, IU/ml } \\
\text { Alanine aminotransferase, IU/ml } \\
\text { Total bilirubin, } \mathrm{mg} / \mathrm{dl} \\
\text { Prothrombin time, INR } \\
\text { Size of liver tumor, cm } \\
\text { Number of liver nodule, } \mathrm{n} \\
\text { Volume of expected remnant liver, \% }\end{array}$ & $\begin{array}{l}120 \pm 13 \\
227 \pm 59 \\
33 \pm 3 \\
43 \pm 3 \\
21 \pm 4 \\
201 \pm 21 \\
2.9 \pm 0.8 \\
4.5 \pm 2.4 \\
1.1 \pm 0.3 \\
73 \pm 6\end{array}$ & $\begin{array}{l}115 \pm 14 \\
236 \pm 78 \\
35 \pm 3 \\
43 \pm 6 \\
25 \pm 4 \\
198 \pm 25 \\
2.8 \pm 0.6 \\
5.3 \pm 1.2 \\
1.2 \pm 0.1 \\
71 \pm 4\end{array}$ & $\begin{array}{l}0.341 \\
0.726 \\
0.096 \\
1.000 \\
0.051 \\
0.732 \\
0.356 \\
0.332 \\
0.317 \\
0.412\end{array}$ \\
\hline Vascular involvement, $n(\%)$ & $\begin{array}{l}\text { Inferior vena cava } \\
\text { Hepatic veins } \\
\text { Portal vein }\end{array}$ & $\begin{array}{l}3(15.0) \\
5(25.0) \\
12(60.0)\end{array}$ & $\begin{array}{l}1(10.0) \\
2(20.0) \\
7(70.0)\end{array}$ & $\begin{array}{l}1.000 \\
1.000 \\
0.702\end{array}$ \\
\hline Location of BTT, $n(\%)$ & $\begin{array}{l}\text { Left/right hepatic duct } \\
\text { Biliary convergence } \\
\text { Common hepatic/bile duct } \\
\text { Intra- and extra-hepatic bile ducts }\end{array}$ & $\begin{array}{l}3(15.0) \\
8(40.0) \\
7(35.0) \\
2(10.0)\end{array}$ & $\begin{array}{l}1(10.0) \\
4(40.0) \\
2(20.0) \\
3(30.0)\end{array}$ & $\begin{array}{l}1.000 \\
1.000 \\
0.675 \\
0.300\end{array}$ \\
\hline
\end{tabular}

B (28.5 [9-54] versus 21.5[6-39] months; $p<.05)$. Among those undergoing thrombectomy alone, group A also had a significantly longer median overall survival than group B (12.8 [6-25] versus 4.5 [2-7] months, $p<.01)$. Among patients undergoing thrombectomy and curative resection, recurrence-free survival was significantly longer in group A than in group B (22.1 [7-50] versus 16.4 [3-35] months, $p<.01)$.

\section{DISCUSSION}

Jaundice is frequently present in HCC patients, at a reported incidence rate of 19 to 40 percent at the time of diagnosis, mainly due to underlying liver cirrhosis, tumor parenchymal involvement, or both (4). Obstructive jaundice caused by BTT, or icteric hepatoma, is relatively uncommon in HCC patients (14). Like vascular invasion, BTT is an unfavorable prognostic factor for HCC patients (7). Thrombectomy and concomitant curative hepatectomy have been attempted to eliminate biliary obstruction and improve overall survival in selected patients (3). However, these patients had a dismal survival outcome with a 5-year survival rate of 28 percent, even after curative liver resection (15). Our present results showed that preoperative TACE could significantly improve the overall survival of HCC/BTT patients, in conjunction with curative resection or tumor thrombectomy alone. To the best of our knowledge, this study is the first report regarding the therapeutic benefit of TACE for HCC/BTT patients.

Portal or hepatic vein thrombosis is usually suggestive of distant metastasis by means of the blood stream and is predictive of a poor survival outcome (16). However, BTT is relatively less progressive due to the space-limiting effect of the bile duct, and BTT is rarely reported to migrate into the gastrointestinal tract (6). Bile duct obstruction caused by BTT, a mix of blood clots and bile sludge, is not necessarily associated with 
Table 2. Operative Outcome and Postoperative Recovery Results

\begin{tabular}{llllr}
\hline & & Group A & Group B & p-value \\
\hline Surgical treatment, $n$ (\%) & Tumor thrombectomy and curative hepatectomy & $12(60.0)$ & $7(70.0)$ & 0.702 \\
& Tumor thrombectomy & $8(40.0)$ & $3(30.0)$ & \\
Liver resection, $n$ (\%) & Right hemihepatectomy & $13(65.0)$ & $6(60.0)$ & 1.000 \\
& Left hemihepatectomy & $5(25.0)$ & $1(10.0)$ & 1.000 \\
& Extended right hemihepatectomy & $1(5.0)$ & & 1.000 \\
& Extended left hemihepatectomy & $1(5.0)$ & $0(0.0)$ & 1.000 \\
Resection of tumor thrombi and liver disease, $n(\%) *$ * & & $3(15.0)$ & $1(10.0)$ & 1.000 \\
Overall operative time, min & & $160 \pm 25$ & $190 \pm 35$ & 0.011 \\
Duration of inflow control, min & & $14.3 \pm 3.6$ & $25.1 \pm 5.1$ & $<0.001$ \\
Intraoperative blood loss, ml & & $150 \pm 35$ & $520 \pm 75$ & $<0.001$ \\
Volume of transfusion, ml & & $100 \pm 40$ & $375 \pm 55$ & $<0.001$ \\
Volume of peritoneal drainage, $m l$ & & $152 \pm 10.2$ & $274 \pm 25.6$ & 0.042 \\
Time to remove peritoneal drain, days & & $5.2 \pm 0.8$ & $8.6 \pm 1.3$ & 0.031 \\
Time to resume anal passage, days & & $4.5 \pm 0.7$ & $5.9 \pm 1.5$ & 0.015 \\
Duration of hospitalization, days & $19.2 \pm 3.2$ & $24.3 \pm 2.9$ & 0.386 \\
\hline
\end{tabular}

* En bloc.

primary liver disease. Thus, obstructive jaundice may occur in BTT and the primary liver disease remains resectable in some patients.

The treatment goal for HCC/BTT should include the elimination of tumor thrombi, the resumption of bile drainage, and curative resection of the primary liver disease if possible. Even tumor thrombectomy alone is expected to be beneficial for the survival of BTT patients, compared with conservative treatment alone. Simple tumor thrombectomy is reported to be comparable to bile duct resection in terms of overall survival (15). Therefore, simple tumor thrombectomy combined with curative liver segmentectomy is preferred for the treatment of HCC/BTT. Intraoperative cholangioscopy can be used to minimize residual tumor thrombi. Exploration of the common bile duct is indicated if dilation of the extrahepatic bile duct is present. The T-tube can be maintained for a longer period in patients undergoing tumor thrombectomy alone, which allows a subsequent cholangioscopic tumor thrombectomy or transcatheter chemotherapy in cases of residual tumor thrombus or recurrence.

The primary procedural risk of tumor thrombectomy is hemobilia, due to the tumor involvement of the bile duct wall. As the bile duct is known to be nourished by the hepatic artery, TACE can interrupt the blood supply to the involved bile duct and downsize and confine the liver tumor. Thus, this neoadjuvant therapy leads to a higher likelihood of curative hepatectomy, reduces blood loss during tumor thrombectomy, decreases surgical morbidity and mortality associated with intraoperative bleeding, and improves patients' survival. HCC patients with complicating BTT exhibited a generally unfavorable survival outcome due to aggravation of hepatic insufficiency, made even worse in cases of concomitant vascular involvement (7). Aggressive surgical treatment has been documented to improve the quality of life and overall survival in these patients. Fukuda et al. (17) reported that curative hepatectomy and thrombectomy combined with hepatic arterial infusion chemotherapy could prolong survival for as much as 12 years. As for HCC patients with vascular tumor thrombosis, preoperative TACE and appropriate surgical treatment is also beneficial for HCC patients with BTT, as shown by our present survival data.

There were some limitations in this study. First, due to the rarity of HCC/BTT the sample size is very small, which weakens the statistical power of the data. Second, because the study was not of a randomized or double-blinded design, the results are subject to some bias. Finally, patients were not further stratified by response to TACE, and it remains unknown whether respondents could benefit more from further curative resection relative to nonrespondents.

\section{CONCLUSIONS}

In conclusion, BTT is highly suspected in cases of jaundiced HCC and generally associated with a poor diagnosis. Tumor thrombectomy, as well as concomitant curative hepatectomy if possible, is beneficial for patients' quality of life and overall 
survival. Preoperative TACE can minimize the risk of hemobilia in tumor thrombectomy and augment the survival benefit of subsequent surgical treatment. The survival benefit of preoperative TACE for HCC patients with complicating BTT shown by the present study warrants further validation in large-scale, prospective, randomized, controlled studies.

\section{CONFLITS OF INTEREST}

We declare that we have no conflict of interest.

\section{REFERENCES}

1. Mehta NFN, Sarkar M, Yao FY. Factors associated with outcomes and response to therapy in patients with infiltrative hepatocellular carcinoma. Clin Gastroenterol Hepatol. 2013;11:572-578.

2. Aldrighetti L, Pulitano C, Catena M, et al. Liver resection with portal vein thrombectomy for hepatocellular carcinoma with vascular invasion. Ann Surg Oncol. 2009;16:1254.

3. Shao W, Sui C, Liu Z, Yang J, Zhou Y. Surgical outcome of hepatocellular carcinoma patients with biliary tumor thrombi. World J Surg Oncol. 2011;9:2.

4. Qin LX, Tang ZY. Hepatocellular carcinoma with obstructive jaundice: Diagnosis, treatment and prognosis. World J Gastroenterol. 2003;9:385391.

5. Liu QY, Lai DM, Liu C, et al. A special recurrent pattern in small hepatocellular carcinoma after treatment: Bile duct tumor thrombus formation. World J Gastroenterol. 2011;17:48174824.

6. Lau W, Leung K, Leung TW, et al. A logical approach to hepatocellular carcinoma presenting with jaundice. Ann Surg. 1997;225: 281-285.
7. Noda T, Nagano H, Tomimaru Y, et al. Prognosis of hepatocellular carcinoma with biliary tumor thrombi after liver surgery. Surgery. 2011;149:371-377.

8. Peng BG, Liang LJ, Li SQ, et al. Surgical treatment of hepatocellular carcinoma with bile duct tumor thrombi. World $J$ Gastroenterol. 2005;11:3966-3969.

9. Yeh CN, Jan YY, Lee WC, Chen MF. Hepatic resection for hepatocellular carcinoma with obstructive jaundice due to biliary tumor thrombi. World J Surg. 2004;28:471-475.

10. Lai EC, Lau WY. Hepatocellular carcinoma presenting with obstructive jaundice. ANZ J Surg. 2006;76:631-636.

11. Mazioti A, Gatselis N.K, Rountas C, et al. Safety and efficacy of transcatheter arterial chemoemboliazation in the real-life management of unresectable hepatocellular carcinoma. Hepat Mon. 2013; 13:e7070.

12. Yoshidome H, Takeuchi D, Kimura F, et al. Treatment strategy for hepatocellular carcinoma with major portal vein or inferior vena cava invasion: A single institution experience. J Am Coll Surg. 2011;212:796-803.

13. Ueda M, Takeuchi T, Takayasu T, et al. Classification and surgical treatment of hepatocellular carcinoma (HCC) with bile duct thrombi. Hepatogastroenterology. 1994;41:349-354.

14. Lin TY, Chen KM, Chen YR, et al. Icteric type hepatoma. Med Chir Dig. 1975;4:267-270.

15. Shiomi M, Kamiya J, Nagino M, et al. Hepatocellular carcinoma with biliary tumor thrombi: Aggressive operative approach after appropriate preoperative management. Surgery. 2001;129:692-688.

16. Peng ZW, Guo RP, Zhang YJ, et al. Hepatic resection versus transcatheter arterial chemoembolization for the treatment of hepatocellular carcinoma with portal vein tumor thrombus. Cancer. 2012;118:47254736.

17. Fukuda S, Okuda K, Imamura M, et al. Surgical resection combined with chemotherapy for advanced hepatocellular carcinoma with tumor thrombus: Report of 19 cases. Surgery. 2002;131:300-310. 